\title{
Extreme-Energy Cosmic Rays: Puzzles, Models, and Maybe Neutrinos
}

\author{
Thomas J. Weiler \\ Department of Physics \& Astronomy, Vanderbilt University, Nashville, TN 37235, USA
}

\begin{abstract}
The observation of twenty cosmic-ray air-showers at and above $10^{20} \mathrm{eV}$ poses fascinating problems for particle astrophysics: how the primary particles are accelerated to these energies, how the primaries get here through the $2.7 \mathrm{~K}$ microwave background filling the Universe, and how the highest-energy events exhibit clustering on few-degree angular scales on the sky when charged particles are expected be bent by cosmic magnetic fields. An overview of the puzzles is presented, followed by a brief discussion of many of the models proposed to solve these puzzles. Emphasis is placed on (i) the signatures by which cosmic ray experiments in the near future will discriminate among the many proposed models, and (ii) the role neutrino primaries may play in resolving the observational issues. It is an exciting prospect that highest-energy cosmic rays may have already presented us with new physics not accessible in terrestrial accelerator searches.
\end{abstract}

\section{INTRODUCTION}

An unsolved astrophysical mystery, now forty years old, is the origin and nature of the extreme energy cosmic ray primaries (EECRs) responsible for the observed events at highest energies, $\sim 10^{20} \mathrm{eV}[1]$. About twenty events at $\sim 10^{20} \mathrm{eV}$ have been observed by five different experiments [2]. The origin of these events is a mystery, for there are no visible source candiates within $50 \mathrm{Mpc}$ except possibly M87, a radio-loud AGN at $\sim 20 \mathrm{Mpc}$, and Cen-A (NGC5128), a radio galaxy at $3.4 \mathrm{Mpc}$, and neither of these is in the direction of any of the observed events [3]. Since the observed events display a large-scale isotropy, many sources rather than one source seem to be required. The nature of the primary particle is also mysterious, because interactions with the $2.73 \mathrm{~K}$ cosmic microwave background (CMB) renders the Universe opaque to nucleons at $E_{\mathrm{GZK}} \sim 5 \times 10^{19} \mathrm{eV}$, and double pair production on the cosmic radio background (CRB) renders the Universe opaque to photons at even lower energies. The theoretical prediction of the end of transparency for nucleons at $E_{\mathrm{GZK}} \sim 0.5 \times 10^{20} \mathrm{eV}$ is the famous "GZK cutoff" [4]. Figure 1 shows a recent compilation of the AGASA data set, clearly extending beyond $E_{\mathrm{GZK}}$.

The main theory challenges in attempting to understand the super-GZK data are: (i) What cosmic source could have accelerated the primary particles to such extremely high energies? and (ii) If the sources are distant ( $\approx 100 \mathrm{Mpc}$ ), then how could their primaries have propagated through the cosmic background radiation without substantial energy loss? The acceleration mechanism either requires a Zevatron accelerator $\left(1 \mathrm{ZeV} \equiv 10^{21} \mathrm{eV}\right)$ [5], distant because such a source could not be missed if it were nearby; or speculative decaying super-massive particles (SMPs) or topological defects (TDs) with massscale $\gtrsim 10^{22} \mathrm{eV}$, clustered nearby; or possibly magnetic monopoles accelerated by the cosmic magnetic fields. From distant Zevatrons, only neutrinos among the known particles can propagate unimpeded to earth. Exploiting this fact are the "Z-burst model," and the strongly- interacting neutrino model. Adding to the drama and mystery at present is the observed large-scale isotropy and small-scale anisotropy. Surely these characteristics hint at a solution to the mystery of origin; protons, nuclei, and magnetic charges bend in cosmic magnetic fields, whereas photons and neutrinos do not. With more data above $E_{\mathrm{GZK}}$, several distinct telltale signatures including the isotropy/anisotropy will allow one to discriminate among the many models proposed for the origin and nature of the EECRs.

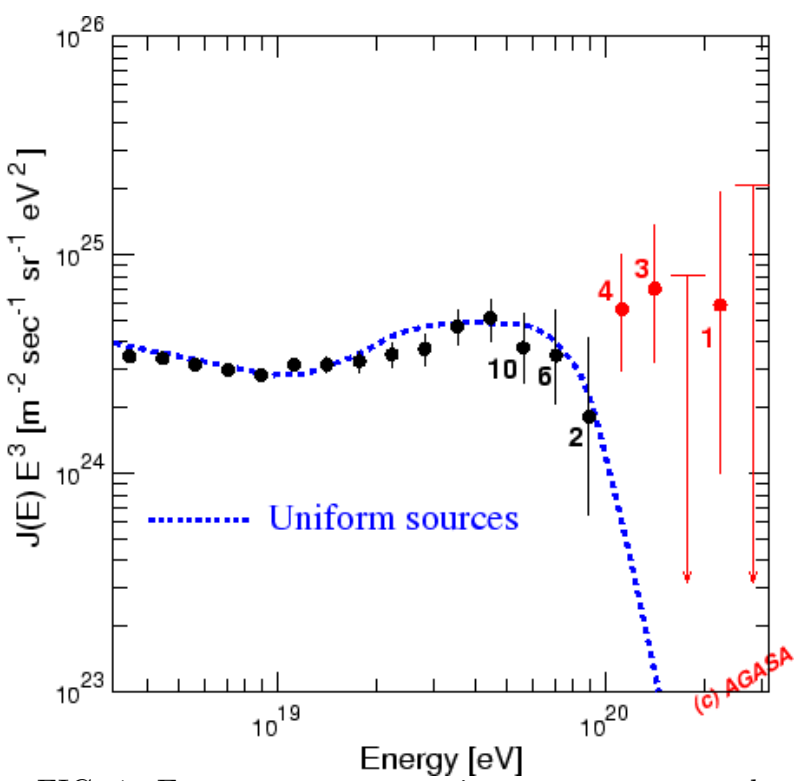

FIG. 1. Extreme-energy cosmic ray spectrum as observed by AGASA. Error bars correspond to $68 \%$ C.L. and the numbers count the events per energy bin. The dashed line revealing the GZK cutoff is the spectrum expected from uniformly distributed astrophysical sources (from the AKENO website $[12])$. 


\section{EVENT CLUSTERING}

One revealing signature is already evident in the existing data sample. This is the pairing of events on the celestial sky. The AGASA experiment has already presented data strongly suggesting that directional pairing is occuring at higher than chance coincidence [6]. Of the 47 published AGASA events above $4 \times 10^{19} \mathrm{eV}, 9$ are contained in three doublets and one triplet with separation angle less than the angular resolution of $2.5^{\circ}$. The chance probability of this clustering occuring in an isotropic distribution is less than 1\%. The chance probability for the triplet alone is only $5 \%$. Of the seven events above $10^{20} \mathrm{eV}$, three are counted among the doublet events. Most recently, AGASA has reported two more events above $E_{\mathrm{GZK}}[7]$. Each aligns in direction with a previous event, reducing further the probability for random clustering to $0.07 \%$ at $3 \sigma$. Comparisons of event directions in a combined data sample of four experiments further supports non-chance coincidences, especially in the direction of the SuperGlactic Plane [8].

Whether the pairing is random or dynamical, we shall also know in the near future. If the pairing turns out to be dynamical, I would argue that neutrinos are a favorite candidate for the primary particles. This is because photons have such a short $(\sim 10 \mathrm{Mpc})$ abosrption length, and protons are bent by cosmic magnetic fields during their extragalactic journey.

If neutrinos are the primaries, they should point back to their sources, thereby enabling point-source astronomy for the most energetic sources of flux at and above $10^{20} \mathrm{eV}$. It was reported [9] that the first five events at $10^{20} \mathrm{eV}$ did in fact point toward extragalactic compact radio-loud quasars, just the class of objects which could accelerate EECRs to ZeV energies via shock mechanisms. With the inclusion of subsequent data, this association is controversial [10]. The jury awaits further evidence.

\section{A. Coincidence or physics?}

A random distribution is obtained by tossing $n$ events randomly into $N \simeq(\Omega / \pi \theta)^{2}=1045(\Omega / 1.0 \mathrm{sr})\left(\theta / 1.0^{\circ}\right)^{-2}$ angular bins, where $\Omega$ is the solid angle on the celestial sphere covered by the experiment and $\theta$ is the bin halfangle. Each resulting event distribution is specified by the partition of the $n$ total events into a number $m_{0}$ of empty bins, a number $m_{1}$ of single hits, etc., among the $N$ angular bins. The probability to obtain a given event topology is [11]

$$
P\left(\left\{m_{i}\right\}, n, N\right)=\frac{1}{N^{n}} \frac{N ! n !}{\prod_{j=0} m_{j} !(j !)^{m_{j}}} .
$$

The variables in the probability are not all independent, as $\sum_{j=1} j \times m_{j}=n$ and $\sum_{j=0} m_{j}=N$. It is useful to use these constraints to rewrite this probability as

$$
P\left(\left\{m_{i}\right\}, n, N\right)=\frac{N !}{N^{N}} \frac{n !}{n^{n}} \prod_{j=0} \frac{\left(\overline{m_{j}}\right)^{m_{j}}}{m_{j} !},
$$

where we have defined

$$
\overline{m_{j}} \equiv N\left(\frac{n}{N}\right)^{j} \frac{1}{j !} .
$$

When $N \gg n \gg 1$, a limit valid for the AGASA, HiRes, Auger and Telescope Array experiments, one finds

$$
P\left(\left\{m_{i}\right\}, n, N\right) \approx \prod_{j=2} \frac{\left(\overline{m_{j}}\right)^{m_{j}}}{m_{j} !} e^{-\overline{m_{j}} r^{j}(j-2) !},
$$

where $r \equiv\left(N-m_{0}\right) / n \approx 1$. The non-Poisson nature of eq. (4) is reflected in the factorials and powers of $r$ in the exponents.

Typical values of effective area $A\left(\mathrm{~km}^{2} \mathrm{sr}\right)$, celestial solid angle $\Omega$, and angular resolution $\theta_{\min }$ for the existing and proposed EECR experiments [12] are shown in the following Table, where the incident flux $F\left(\geq E_{\mathrm{GZK}}\right)=$ $10^{-19} \mathrm{~cm}^{-2} \mathrm{~s}^{-1} \mathrm{sr}^{-1}$ has been used to estimate the number of events $(n / \mathrm{yr})$ above $E_{\mathrm{GZK}}=5 \times 10^{19} \mathrm{eV}$.

\begin{tabular}{|c|c|c|c|c|}
\hline & AGASA & HiRes & Auger/TA & EUSO/OWL \\
\hline $\mathrm{km}^{2} \mathrm{sr}$ & 150 & 800 & $6 \times 10^{3}$ & $3 \times 10^{5}$ \\
\hline$n / \mathrm{yr}$ & 5 & 30 & 200 & $10^{4}$ \\
\hline$\Omega(\mathrm{sr})$ & 4.8 & 7.3 & 4.8 & $4 \pi$ \\
\hline$\theta_{\min }$ & $3.0^{\circ}$ & $0.5^{\circ}$ & $1.0^{\circ}$ & $1.0^{\circ}$ \\
\hline
\end{tabular}

In Figure 2 are shown inclusive probabilities for the Auger experiment with 100 events, as determined by formulae eq. (4). Note that "inclusive probability" means the stated number of $j$-plets plus any other clusters. The 8-doublet probability is extremely sensitive to the angular binning; observation of a flatter dependence on angular bin-size could signal a non-chance origin for the clustering. The observation of two triplets with angular binning of less than $2^{\circ}$ would constitute 3 -sigma evidence for cosmic dynamics. The same conclusion would hold if a quadruplet within $2.5^{\circ}$ is observed among the first 100 Auger events.

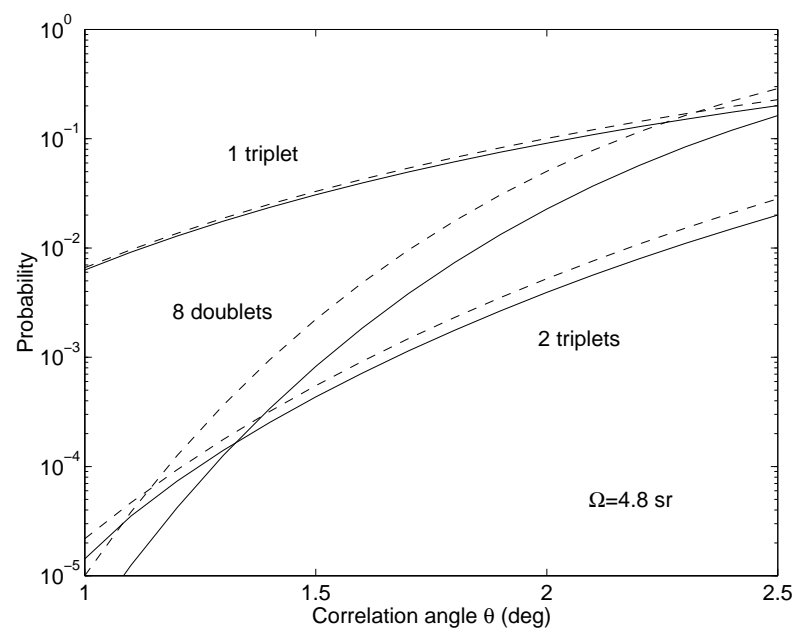


Figure 2: Inclusive probabilities for various clusters in a 100 event sample at Auger. The solid line is the exact result, the dashed line is the Poisson approximation

Without some modification, our analytic formula may not be directly relevant to HiRes data. Clear, moonless nights are required for detection of atmospheric fluorescence, and summer nights $(\sim 18 \mathrm{hrs}$ right ascension $)$ are effectively $40 \%$ shorter than winter nights $(\sim 6 \mathrm{hrs}$ right ascension) for monocular HiRes [13]. Accordingly, the HiRes efficiency versus Galactic longitude varies significantly, roughly as $N(R A)=\bar{N}(1+\epsilon \sin (2 \pi R A / 24 \mathrm{hrs}))$, with $\epsilon \approx 0.25$. One must ask whether this sinusoidallyvarying efficiency invalidates the analytic approach with its assumption of a constant efficiency. In the Monte Carlo approach there is an easy method to generate "background" data sets with the experimental efficiency properly incuded - one randomly permutes the RA coordinates of the real data to remove any dynamical correlation among the events. Fortunately, there is also a simple method to estimate the efficiency correction to an analytic Poisson distribution, if one assumes that although $N$ and $n$ vary with right ascension, $\xi \equiv n / N$ is constant. Then it is easy to show that the Poisson distribution

$$
P(j, m)=\left(\frac{N \xi^{j}}{j !}\right)^{m} \frac{1}{m !} e^{-N \xi^{j} / j !}
$$

is corrected upwards by the factor

$$
\mathcal{I}=\frac{1}{2 \pi} \int_{0}^{2 \pi} d \phi(1+\epsilon \sin (\phi))^{m} e^{-\bar{N} \epsilon \sin (\phi) \xi^{j} / j !} .
$$

For $\frac{\epsilon \xi^{j-2}}{j !} \frac{n^{2}}{\bar{N}} \ll 1$, the exponential is near unity and the correction has a closed form, $\mathcal{I}_{m} \approx\left(1-\epsilon^{2}\right)^{m / 2} P_{m}\left(\frac{1}{\sqrt{1-\epsilon^{2}}}\right)$, with $P_{m}$ a Legendre polynomial. As a series, the correction is

$$
\mathcal{I}_{m}=2^{-m} \sum_{k=0}^{[m / 2]} \frac{(-)^{k}(2 m-2 k) !}{k !(m-k) !(m-2 k) !}\left(1-\epsilon^{2}\right)^{k} .
$$

In particular, for $n^{2} \ll \bar{N}$, we have $\mathcal{I}_{1}=1$ independent of $\epsilon, \mathcal{I}_{2}=1+\epsilon^{2} / 2,=1.03$ for HiRes, $\mathcal{I}_{3}=1+3 \epsilon^{2} / 2,=$ 1.09 for HiRes, and $\mathcal{I}_{4}=1+3 \epsilon^{2}+3 \epsilon^{4} / 8,=1.189$ for HiRes. The lesson is that HiRes efficiency corrections appear negligible for small numbers of clusters, and we may proceed with our analytic analysis for the HiRes experiment, for which about 20 events at $10^{20} \mathrm{eV}$ are expected when the first full year's data is analyzed. We display in Figure (3) the inclusive probabilities for one or more, two or more, and three or more doublets; and one or more triplets, over a range of angular binning.

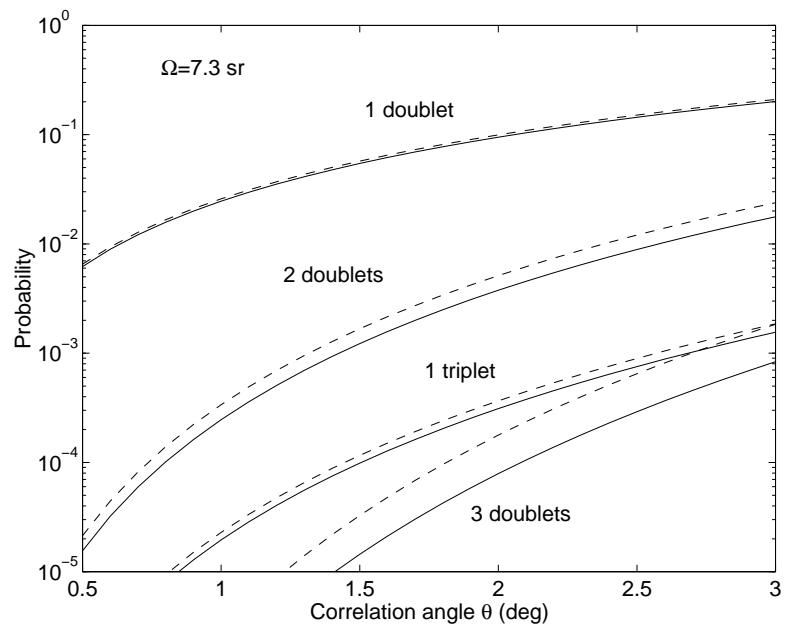

Figure 3: Inclusive probabilities for various clusters, given 20 events at HiRes. The solid line is the exact result, the dashed line is the Poisson approximation.

Note that for all except the 3 doublet configuration, the Poisson approximation using the mean values in Eq.(3) provides an estimate good to within $50 \%$ of the nonapproximate form; for the (much suppressed) 3 doublet configuration, it overestimates the probability by about a factor of 3 in much of the angular region. For angular binning tighter than $2^{\circ}$, an observation of two doublets among the first 20 events has a chance probability of less than $0.5 \%$. Thus the observation of this topology could be construed as evidence (at the $3 \sigma$ level) for clustering beyond statistical. The observation of a triplet within $\leq 3^{\circ}$ has a random probability of less than $10^{-3}$, and hence observation of such a triplet would most likely signify clustered or repeating sources, or magnetic focusing effects. With the accumulation of 40 events (not shown in the figure), the appearance of two doublets has a probability of less than $0.5 \%$ for a correlation angle of $1^{\circ}$ or less. This illustrates how the good angular resolution of HiRes may be used to detect non-statistical clustering with only a few observed clusters.

Projected event rates for the EUSO/OWL/AW experiments present a pleasant problem for our analytic formula. In the case where $n>N \gg 1$, relevant for the EUSO/OWL/AW experiments after a year or more of running, higher $j$-plets are common and the distribution of clusters can be rather broad in $j$. From eq. (3) we have $\left(\overline{m_{j}} / \overline{m_{j-1}}\right)=(n / j N) \sim\left(\pi n \theta^{2} / j \Omega\right)$. Already at $j=1(2)$, Stirling's approximation to $j$ ! is good to $8 \%(4 \%)$, and so for $j \geq 1$ we may approximate $\overline{m_{j}} \approx\left(N^{3} / 2 \pi e n\right)^{\frac{1}{2}}(e n / j N)^{j+\frac{1}{2}}$. Extremizing this expression with respect to $j$, one learns that The most populated $j$-plet occurs near $j \sim n / N$. Combining this result with the broad distribution expected for large $n / N$, one expects clusters with $j$ up to several $\times \frac{n}{N}$ to be common in the EUSO/OWL/AW experiments. Probabilities and meaning for frequent, large clusters are somewhat difficult to assess numerically. 
The random distributions displayed in figs. (2) and (3) are expected from some models, such as randomlysituated decaying super-massive particles (SMPs), or charged-particle or magnetic monopole primaries with directions randomized by incoherent cosmic magnetic fields. A complementary approach to the random probabilities shown here is to consider specific source models generating non-random angular distributions. Steps along this line of inquiry have recently been taken [14]. Future progress in the field will involve comparisons of the random and non-random model predictions with the data.

\section{MODELS FOR SUPER-GZK EVENTS}

The conjectured origins of the super-GZK particles fall into four basic categories. These are (i) nearby accelerators, (ii) exotic primaries, (iii) exotic physical law, and (iv) neutrino primaries. Other reviews [1] have emphasized the first three categories (especially (i) and (ii)), so here we will be brief with those, and put more emphasis on the neutrino-primary option.

\section{A. Nearby accelerators}

Several types of sources have been proposed to exist within our Galactic halo. These include highly-ionized relativistic dust grains, Galactic supershocks, young neutron stars, magnetars (highly magnetized pulsars), decaying SMPs with GUT masses of order $10^{15} \mathrm{GeV}$ or with inflation-motivated masses of order $10^{13 \pm 1} \mathrm{GeV}$, topological defects [15] such as strings, Q-balls and vortons, and annihilating monopole-antimonopole bound states (monopolonium) [16]. For rare sources emitting charged particles, such as magnetars, it is necessary to postulate that the primaries are iron nuclei to ease the acceleration requirement and to isotropize the flux in our galactic magnetic field. It is intriguing that the observed magnitude of $\mathrm{CMB}$ fluctuations fixes the reheat temperature following inflation to $10^{13 \pm 1} \mathrm{GeV}$, which allows gravitational and thermal production of TDS and/or SMPs of just this mass (if they exist) [17]; this mass scale is just right for producing $10^{20}$ secondary particles via decay.

For some TD models, dimensional arguments and scaling laws seem to give an emission rate to short-lived SMPs consistent with the observed EECR rate without tuning exotic parameters. However, for most decaying sources such as SMPs, TDs, and monopolonium, it is necessary to tune the lifetime to be longer but not too much longer than the age of the Universe in order to maintain an appreciable secondary particle emission rate today. Discrete gauged symmetries [18] or hidden sectors [19] are introduced to stabilize the heavy particle. Then rather esoteric physics is needed to break the new symmetry super-softly to maintain the long lifetime. High- dimension operators, wormholes [20], and instantons [21] bibiteminstanton have been invoked for this purpose.

Models with sources mainly in the Galactic plane are disfavored by the lack of any observed planar asisotropy in the data. Models with sources clustered in the Galactic halo predict a dipole enhancement in the direction of the Galactic center [22], which will be tested by the Auger Observatory in a few years.

Possible sources outside our halo, but still relatively nearby, include the radio-loud quasar M87 at 18 Mpc [23], the similar Cen-A at 3.4 Mpc [24], rare nearby GRBs, and now-dormant rare AGNs (also called massive dark objects - MDOs). These sources are few at best, and strong magnetic fields must be postulated to isotropize and/or confine their emissions. In the case of GRBs, the identification of the red-shift of their host galaxies at typically $\gtrsim 1$ renders a local occurence of a GRB highly improbable [25].

Finally, primordial black holes (PBHs) have also been suggested, but any sensible initial mass spectrum is unable to provide a sufficient number of PBHs in the final stage of decay today.

\section{B. Exotic primaries}

The GZK cutoff can be raised by simply postulating a primary hadron slightly heavier than the proton. The reason is kinematical - the cutoff energy varies as the square of the mass of the first excited resonant state. For this reason, Farrar has proposed that light supersymmetric baryons, made from a light gluino plus the usual quarks and gluons, may be the primary EECRs [26]. Such a scenario renders the source-energetics issue even more challenging. In any event, terrestrial experiments seem to have recently closed the window on a possible light gluino.

Another interesting possibility for the primary EECR is the magnetic monopole [27,28]. Any breaking of a semisimple gauge symmetry which occurs after inflation and which leaves unbroken a $U(1)$ symmetry group may produce an observable abundance of magnetic monopoles. The monopole mass is expected to be $\sim \alpha^{-1}$ times the temperature $T_{c}$ of the symmetry breaking. At the time of the phase transition, roughly one monopole or antimonopole is produced per correlated volume. The resulting monopole number density today is

$$
n_{M} \sim 10^{-19}\left(T_{c} / 10^{11} \mathrm{GeV}\right)^{3}\left(l_{H} / \xi_{c}\right)^{3} \mathrm{~cm}^{-3},
$$

where $\xi_{c}$ is the phase transition correlation length, bounded from above by the horizon size $l_{H}$ at the time of the transition. In a second order or weakly first order phase transition, the correlation length is comparable to the horizon size. In a strongly first order transition, the correlation length is considerably smaller than the horizon size. 
The kinetic energy imparted to a magnetic monopole on traversing a magnetic field $B$ is $E_{K} \sim g B \xi$, where $g=e / 2 \alpha$ is the magnetic charge according to the Dirac quantization condition, and $\xi$ specifies the field's coherence length. Given the magnitude and coherence length data for the cosmic magnetic fields, monopole kinetic energies in the range $10^{20}$ to $10^{23} \mathrm{eV}$ are expected; the acceleration problem is naturally solved. Monopoles with $M \lesssim 10^{14} \mathrm{GeV}$ should be relativistic, and carry the appropriate energy to qualify as candidates for the EECR primaries [28,29]. Within field theory there exist many possibilities for an intermediate unification scale and intermediate-mass monopoles.

The propagation problem is also naturally solved. The scattering cross-section for the monopole on the $3 \mathrm{~K}$ and diffuse photon backgrounds is just classical Thomson, valid even for strong coupling: $\sigma_{\mathrm{T}}=8 \pi \alpha_{M}^{2} / 3 M^{2} \sim$ $2 \times 10^{-43}\left(M / 10^{10} \mathrm{GeV}\right)^{-2} \mathrm{~cm}^{2}$. The resulting mean free path for inverse Compton scattering is many orders of magnitude larger than the Hubble size of the Universe.

The relativistic monopole flux is simply

$$
F_{M}=c n_{M} / 4 \pi \sim 2 \times 10^{-19}\left(M / 10^{10} \mathrm{GeV}\right)^{3}\left(l_{H} / \xi_{c}\right)^{3}
$$

per $\mathrm{cm}^{2}$-s-sr, which compares favorably with the integrated flux above $10^{20} \mathrm{eV}, F_{\text {data }}\left(>10^{20} \mathrm{eV}\right) \sim 2 \times$ $10^{-20} \mathrm{~cm}^{-2} \mathrm{~s}^{-1} \mathrm{sr}^{-1}$, and is comfortably below Parker's upper bound $F_{\text {Parker }}=10^{-15} \mathrm{~cm}^{-2} \mathrm{~s}^{-1} \mathrm{sr}^{-1}$ for a cosmic monopole flux.

Signatures for EECR monopoles in our atmosphere and in ice are discussed at length in [29]. Of particular interest as a model for the super-GZK primaries is the "baryonic monopole" [30,29]. It is a bound state of three colored monopoles, confined by chromomagntic strings. Chromomagnetic strings can stretch when excited, but cannot break into quark-antiquark pairs. On first interaction in the atmosphere, the baryonic monopole of mass $M$ and energy $E=\gamma M$ stretches to create a huge geometrical cross-section of order $\gamma / \Lambda_{\mathrm{QCD}}^{2} \sim\left(\gamma / 10^{6}\right) \times$ $10^{7} \mathrm{mb}$. Consequently, nearly all of the initial monopole energy is transferred to the atmospheric shower in a very short distance. A recent simulation of the baryonicmonopole showed good agreement with the lateral muon and hadron content of the highest-energy Yakutsk event, but less than good agreement with the longitudinal profile of the Fly's Eye event [31].

Any confirmed directional pairing of events would appear difficult to achieve with the monopole model. Also, in the context of a model of the Galactic magnetic field, it has been shown that some memory of the local spiral arm direction, and an energy spectrum flatter than the observed one, are expected in the data if monopoles are the primaries [32]. Although the directional criterion may not survive inclusion of extragalactic fields, the flatness of the spectrum probably does.

\section{Exotic physical laws}

The most remarkable proposals posit a breakdown of Lorentz invariance [33] or a breakdown of general relativity above some high scale. In the case of broken GR, it could be spacetime fluctuations (expected in a theory of quantum gravity) wiggling the on-shell dispersion relation [34] or appearing as $1 / M_{P}$ operators which alter the physics. String theory provides motivation for GR-breaking at a possibly lower scale, the string scale $M_{S}$. Certainly the energy window of EECRs $\left(10^{20} \mathrm{eV} / M_{P} \sim 10^{-8}\right)$ is beyond that of terrestrial accelerators, and so ripe for speculation on new high-energy physics.

Signatures of models with no photo-pion production above $E_{\mathrm{GZK}}$ include the absence of a proton pile-up below $E_{\mathrm{GZK}}$, the absence of a cosmogenic neutrino flux, and possibly undeflected pointing of the primary back to its source.

\section{Neutrino primaries}

Turning to the possibility that the primaries may be neutrinos, one encounters an immediate obstacle: the SM neutrino cross-section is down from that of an electromagnetic or hadronic interaction by six orders of magnitude. This implies a low air-shower rate, and an accumulation of events at low altitudes ("penetrating" events) where the target density is highest. On the other hand, the neutrino-primary hypothesis is supported by the observed clustering discussed earlier. Two solutions to the small cross-section problem for primary neutrinos have been proposed.

\section{Neutrino annihilation to $Z$-bursts}

Here it is proposed that the primary particles which propagate across cosmic distances above the GZK cutoff energy are neutrinos, which then annihilate with the cosmic neutrino background (CNB) within the GZK zone $\left(D<D_{\mathrm{GZK}} \sim 50 \mathrm{Mpc}\right)$ to create a "local" flux of nucleons and photons above $E_{\mathrm{GZK}}$, as shown in Fig. (4). It was noted many years ago that a cosmic ray neutrino arriving at earth from a cosmically distant source has an annihilation probability on the relic-neutrino background of roughly $3.0 h_{65}^{-1} \%$ (neglecting cosmic expansion) [35]. The probability for a neutrino with resonant energy to annihilate to a $Z$-burst within distance $D_{\mathrm{GZK}}$ is then $2.5 \times 10^{-4}$ for a homogeneous CNB [36]. The annihilation rate depends upon the $\mathrm{CNB}$ density, reliably predicted in the mean by Big Bang cosmology, and on the Standard Model (SM) of particle physics. The local annihilation rate is larger if our matter-rich portion of the Universe clusters neutrinos [36,37], or if there is an intrinsic CP-violating $\nu-\bar{\nu}$ asymmetry [38]. 


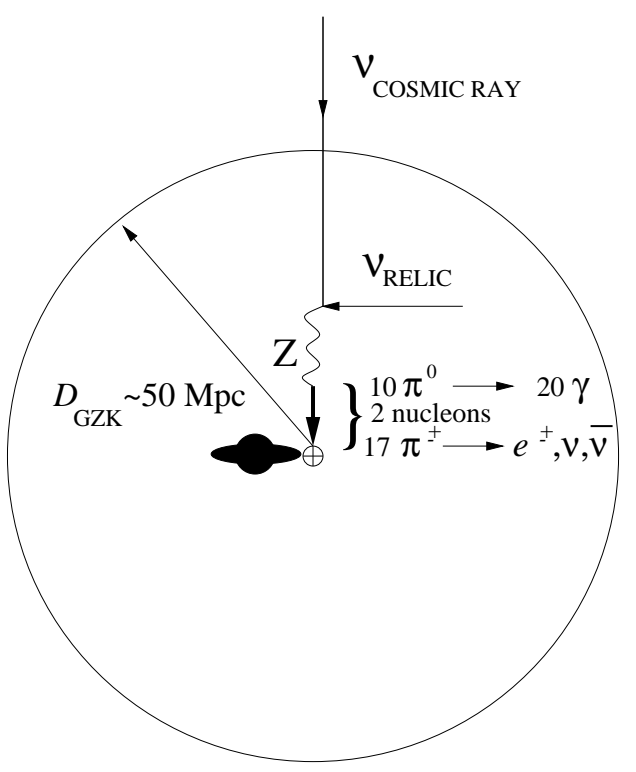

Figure 4: Schematic diagram showing the production of a $Z$-burst resulting from the resonant annihilation of a cosmicray neutrino on a relic (anti)neutrino. If the $Z$-burst occurs within the GZK zone ( $\sim 50$ to $100 \mathrm{Mpc}$ ) and is directed towards the earth, then photons and nucleons with energy above the GZK cutoff may arrive at earth and initiate super-GZK air-showers.

Each resonant neutrino annihilation produces a $Z$ boson with a $70 \%$ branching ratio into hadrons known to include on average about one baryon-antibaryon pair, seventeen charged pions, and ten neutral pions [39]. The ten $\pi^{0}$ 's decay to produce twenty high-energy photons. For $m_{\nu}$ in the range $\sim 0.1$ to $2 \mathrm{eV}$, the energy in this resonant " $Z$-burst" is fortuitously situated sufficiently above $E_{\mathrm{GZK}}$ at

$$
E_{\nu}^{R}=M_{Z}^{2} / 2 m_{\nu}=4\left(\mathrm{eV} / m_{\nu}\right) \times 10^{21} \mathrm{eV}
$$

so as to produce photons and nucleons with energies exceeding $E_{\mathrm{GZK}}{ }^{*}$ The mean energies of the $\sim 2$ baryons and $\sim 20$ photons produced in the $\mathrm{Z}$ decay are easily estimated. Distributing the Z-burst energy among the mean multiplicity of 30 secondaries in Z-decay [39], one has

$$
\left\langle E_{p}\right\rangle \sim \frac{E_{R}}{30} \sim 1.3\left(\frac{\mathrm{eV}}{m_{j}}\right) \times 10^{20} \mathrm{eV} .
$$

The photon energy is further reduced by an additional factor of 2 to account for their origin in two-body $\pi^{0}$ decay:

$$
\left\langle E_{\gamma}\right\rangle \sim \frac{E_{R}}{60} \sim 0.7\left(\frac{\mathrm{eV}}{m_{j}}\right) \times 10^{20} \mathrm{eV} .
$$

\footnotetext{
${ }^{*}$ The resonant-energy width is narrow, reflecting the narrow width of the Z-boson: at FWHM $\Delta E_{R} / E_{R} \sim \Gamma_{Z} / M_{Z}=3 \%$.
}

Even allowing for energy fluctuations about mean values, it is clear that in the Z-burst model the relevant neutrino mass cannot exceed $\sim 2 \mathrm{eV}$. On the other hand, the neutrino mass cannot be too light or the predicted primary energies will exceed the observed event energies. In this way, one obtains the approximate $0.1 \mathrm{eV}$ lower limit on the neutrino mass, when allowance is made for an order of magnitude energy-loss for those secondaries traversing 50 to $100 \mathrm{Mpc}$.

The challenging issue of how experiments might actually determine the absolute neutrino mass is discussed in [40].

If the $Z$-burst points in the direction of earth and occurs within the GZK distance, then one or more of the photons and nucleons in the burst may initiate a superGZK air-shower at earth [36,37]. For a sufficient cosmic neutrino flux, the hypothesis successfully explains the observed air-showers above $E_{\mathrm{GZK}}$. Comparisons of the model predictions to super-GZK data are available in [41].

The existence of neutrino mass in the desired range seems nearly guaranteed from the tritium decay upper bound [42] and the lower bounds inferred from the terrestrial neutrino oscillation experiments. The simplest explanation for the atmospheric neutrino results is neutrino oscillations driven by a mass-squared difference of $\delta m_{\text {atm }}^{2} \sim 3 \times 10^{-3} \mathrm{eV}^{2}$ [43], which implies a neutrino mass of at least $0.05 \mathrm{eV}$. Also, the recent LSND measurement appears to indicate a mass-squared difference $\delta m_{\mathrm{LSND}}^{2} \gtrsim 0.2 \mathrm{eV}^{2}[44]$, from which one deduces a neutrino mass of at least $0.5 \mathrm{eV}$. From these lower bounds on neutrino mass, one gets upper bounds on the $Z$-burst energy of $10^{23}$ and $10^{22} \mathrm{eV}$, respectively, just right for extending the air-shower spectrum an order of magnitude or two beyond the GZK cutoff!

A considerable cosmic neutrino flux above $E_{\mathrm{GZK}}$ is required for the $Z$-burst hypothesis to successfully explain the super-GZK events. The requirement is that the product of the resonant energy times the neutrino flux at the resonant energy per flavor, times the annihilation probability within the GZK zone (which may be as large as $0.025 \%$ to $1 \%$ due to neutrino clustering), times the photon and nucleon multiplicity per burst $(\sim 20)$, is comparable to the observed flux at $10^{20} \mathrm{eV}$. The resulting requirement on the neutrino flux is roughly $E_{R} F_{\nu_{j}}\left(E_{R}\right) \sim 10^{-18.5 \pm 1} / \mathrm{cm}^{2} / \mathrm{s} / \mathrm{sr}$. Such a neutrino flux at $E \sim 10^{22} \mathrm{eV}$ is directly measureable in a teraton ( $10^{12}$ ton) detector like EUSO/OWL/AirWatch, and possibly in a search for radio pulses produced by high energy neutrinos penetrating a small column-density of matter in the limb of the moon [45].

While certainly large, this required neutrino flux violates no existing limits. It has been pointed out [46] that this flux cannot extrapolate as $E^{-2}$ to $10^{17} \mathrm{eV}$, for then it would violate the Fly's Eye bound arising from nonobservation of penetrating horizonal (i.e., neutrino induced) air showers at that energy. It has also been pointed out 
[47] that local neutrino clustering is required to avoid generating a density of $30 \mathrm{MeV}$ to $100 \mathrm{GeV}$ photons in excess of the EGRET experimental bound [48], from distant Z-bursts undergoing electromagnetic cascading. The extreme-energy neutrino flux implied by the $Z$-burst model probably requires unusual source dynamics [49]. Among the reasons to hope that Nature obliges is that resonant neutrino annihilation provides the best hope at present to actually measure the relic neutrino density.

\section{Strong $\nu$ Cross-section at $\gtrsim E_{\mathrm{GZK}}$}

It is interesting and suggestive that the observed EECR flux beyond $E_{\mathrm{GZK}}$ is well matched by the flux predicted for cosmogenic neutrinos. This is not a complete coincidence. With the GZK cutoff, any continued nucleon flux beyond $E_{\mathrm{GZK}}$ is degraded in energy, photoproducing pions which in turn decay to produce cosmogenic neutrinos. The number of produced neutrinos compensates for their lesser energy, with the result that the neutrino flux matches well to the observed super-GZK flux. One may entertain the notion that the cosmogenic neutrinos are the super-GZK primaries, and that these neutrinos acquire a strong cross-section at $\sim 10^{20} \mathrm{eV}$.

Limits on the strength of the neutrino cross-section at $10^{20} \mathrm{eV}$ can be inferred from existing data. Heuristically, one argues as follows. The GZK process ensures that there is a flux of cosmogenic neutrinos at $10^{20} \mathrm{eV}$, with an easily calculated flux. If the neutrino cross-section were weak, an experiment looking for penetrating airshowers initiated by the cosmogenic neutrinos would see nothing. If the cross-section were strong enough, the neutrino could not penetrate the atmosphere at all. So the fact that the Fly's Eye experiment saw no penetrating showers tells us that the neutrino cross-section is either strong or weak; the mid-range is excluded. The vertical depth of our atmosphere is $x_{\mathrm{v}}=1033 \mathrm{~g} / \mathrm{cm}^{2}$, and the horizontal depth $x_{\mathrm{h}}$ is about 36 times greater. In terms of the mean free path $\lambda$ of a particle with cross-section $\sigma$, one has $x_{\mathrm{v}} / \lambda=\sigma / 1.6 \mathrm{mb}$, and $x_{\mathrm{h}} / \lambda=\sigma / 44 \mu \mathrm{b}$. Thus, an estimate of the excluded cross-section is $\sim 40 \mu \mathrm{b}$ to $1 \mathrm{mb}$. A more careful calculation has been performed, with the result that $\sim 20 \mu \mathrm{b}$ to $1 \mathrm{mb}$ is excluded [50]. Hypothetical high-energy neutrino cross-sections in excess of a mb remain viable.

The idea that neutrinos, indeed, all particles, may have a strong interaction at a high but observable energy scale is not new [51]. However, some recent ideas concerning new interactions relate well to the $10^{20} \mathrm{eV}$ scale. One idea is that leptons are bound states of dual QCD gluons, which reveal themselves just above the electroweak (EW) scale at parton-parton $\sqrt{s} \sim \mathrm{TeV}[52]$. Another idea is that grand unification occurs precociously at $\sqrt{s} \sim \mathrm{TeV}$, because of extra dimensions or other reasons, and a neutrino above this threshold becomes strongly-interacting via leptoquark resonances [53]. A third idea is that the exchange of a towers of Kaluza-Klein (KK) modes from extra compactified dimensions lead to a strong neutrino cross-section above $\sqrt{s} \sim \mathrm{TeV}$ [54]. In all three cases, it is the combination of a low $\sim \mathrm{TeV}$ scale for radically new physics and a quickly rising spectrum of new states (possibly increasing exponentially, $\rho \sim e^{\sqrt{s / s_{0}}}$ ) that provides a rapid turn-on of a strong cross-section for the neutrino. Through unitarity, the new threshold at $\sim \mathrm{TeV}$ has consequences for cross-sections at lower energies [55], but they are not dramatic.

The KK exchange model may fail [56] in that the KK modes couple to neutral currents, and the scattered neutrino carries away $90 \%$ of the incident energy per interaction, thereby elongating the shower profile. But if the neutrino cross-section can be made large enough, $\gtrsim 20 \mathrm{mb}$, then multiple scattering within a nucleus will effect a sufficiently large energy transfer and save the model [57]. Independent of the neutral current issue, the dual QCD and TeV-scale unification models seem to provide viable explanations of the super-GZK data. However, a recent calculation for the rate of rise of the low-scale unification cross-section in a string context is not encouraging [58].

Signatures for these models include directional pointing back to the EECR source, longitudinal shower profiles differing somewhat from those of a proton or a gamma, and a strong correlation between observed energies and zenith angle. The latter signature should show an inverse proportionality between the neutrino-air interaction length and the rising (with energy) neutrino crosssection.

\section{MODEL SIGNATURES}

There are several telltale discriminators to be sought in higher statistics data. These will eventually eliminate most (perhaps all!) of the models so far proposed for the super-GZK events. We list some discriminating signatures and discuss them.

\section{A. Small-scale anisotropies and pointing}

The discriminatory power of small-scale clustering was discussed already in $\S I I A$. Here we add some detail to the discussion. In traversing a distance $D$, a charged particle interacting with magnetic domains having coherence length $\lambda$ will bend through an energy-dependent angle ${ }^{\dagger}$

\footnotetext{
${ }^{\dagger}$ On average, half of the interactions of a super-GZK nucleon with the $\mathrm{CMB}$ change the isospin. At energies for which $c \tau$ of the neutron is small compared to the interaction mfp of $\sim 6 \mathrm{Mpc}$, the neutron decays back to a proton with negligi-
} 


$$
\delta \theta \sim 0.5^{\circ} \times \frac{Z B_{n G}}{E_{20}} \sqrt{D_{\mathrm{Mpc}} \lambda_{\mathrm{Mpc}}} .
$$

Here $B_{n G}$ is the magnetic field in units of nanogauss, $E_{20}$ and $Z$ are the particle energy in units of $10^{20} \mathrm{eV}$ and charge, and the lengths $D$ and $\lambda$ are given in units of Mpc. It is thought likely that coherent extragalactic fields are nanogauss in magnitude [59], in which case super-GZK primaries from $\lesssim 50$ Mpc will typically bend only a few degrees (but note that protons at $10^{19} \mathrm{eV}$ will bend through $\left.\sim 30^{\circ}\right)$. Thus, local models either postulate many invisible sources isotropically-distributed with respect to the Galaxy to provide the roughly isotropic flux observed above $E_{\mathrm{GZK}}$, or postulate a large extragalactic magnetic field to isotropize over our Northern Hemisphere the highest-energy particles from a small number of sources [60]. Among the latter category, some models postulate helium or iron nuclei as the primaries, to increase the bending by the charge factors 2 and 26 , respectively. For those models invoking randomly distributed, decaying super-massive particles (SMPs) [61] or topological defects (TDs) [62] as sources, and models invoking a large magnetic field with considerable incoherent component, one may expect a nearly chance distribution of observed events on the sky. However, there may be some clustering even in these models, due to possible small-scale density fluctuations in the local SMP or TD distributions [63], or due to possible caustics in the projection of large-scale extragalactic magnetic fields on our sky (assuming the incoherent magnetic fields are sufficiently small) [64]. In the SMP and TD models, a high photon fraction in the primary composition further enhances clustering possibilities.

From the point of view of opening a new window to astronomy, those models in which the primaries do point back to their distant, active sources are the most interesting. These models are few in number. They are the Z-burst model, the strongly-interacting neutrino model, and the quantum-gravity/LI-violating models.

\section{B. Large-scale anisotropies}

On large scales, one seeks associations of the CR directions with the Galactic halo (to be revealed by a dipole anisotropy favoring the direction of the Galactic center) or the local galactic magnetic field, with matter distributions in nearby galactic or super-galactic clusters such Virgo, or with possible large coherent galactic or extragalactic magnetic fields [65]. For large-scale

ble energy loss and the bending-angle formula is unchanged. However, at the energy $6 \times 10^{20}, c \tau$ for the neutron is comparable to the interaction $\mathrm{mfp}$, so at higher energies the nucleon bending-angle is reduced by $\lesssim 2$. studies, the Southern hemisphere Auger experiment will prove invaluable for several reasons. It offers coverage of potential sources and matter distributions, and galactic and extragalactic magnetic fields, not available from the North. Moreover, it offers a view of our Galactic center which will provide a North-South dipole discriminator for or against a halo-centered population of sources such as magnetars or halo-bound SMPs. Southern Auger will also discriminate the M87-source model [23] wherein EECRs are channeled by a hypothesized galactic magnetic wind into the Northern hemisphere, and the Cen-A source model [24] which also yields a dipole anisotropy. Of course, an orbiting experiment with $4 \pi$ vision like EUSO/OWL will be an even better instrument for multipole analysis.

\section{Energy-direction-time correlations}

Because bending of charged particle trajectories by intervening magnetic fields increases as particle energy decreases, one may learn about the strength and geometry of extragalactic fields from relative time delays and angular correlations of particles from a common source. One may also learn about the source. Quantitatively, the increase in path length due to bending leads to a relative increase in travel time of $\delta t / t \sim(\delta \theta)^{2}$, for small bending angle. Adding the contributions from the coherent magnetic domains then yields

$$
\delta t \sim 300 D_{\mathrm{Mpc}}\left(\frac{Z B_{n G} \lambda}{E_{20}}\right)^{2} \mathrm{yrs}
$$

for the time delay. The time separation at earth is obtained by taking differences in eq. (14); to first order in $\delta E$ it is already large:

$$
t_{1}-t_{2} \sim 600 D_{\mathrm{Mpc}}\left(\frac{Z B_{n G} \lambda}{E_{20}}\right)^{2}\left(\frac{\delta E}{E_{20}}\right) \mathrm{yrs} .
$$

The correlation in energy and time becomes even more significant when it is remembered that the higher energy primary has an even higher mean energy in transit, before losses on the $2.7 \mathrm{~K}$ background.

Surprisingly, one AGASA event-pair has the higherenergy $1.06 \times 10^{20}$ primary arriving about 3 years after a $0.44 \times 10^{20}$ primary. Assuming these primaries originate form a common source, a possible explanation is that their source has a duration of at least $3 /(1+z)$ years (the red-shift factor is due to time-dilation). Such a long-lived source does not occur in one-time burst models (e.g. GRBs). It may occur in decay models (e.g. SMPs, TDs, monopolonium) if sub-clustering exists on smallangular scales withing halo clusters [66]. A recent paper [67] notes that such sub-clusters may actually be observable as micro-lenses for stars and background galaxies. Counter-intuitive reverse pairing with the earlier arrival time for the lower energy charged-primary can also occur in certain magnetic field configurations, as shown in [68]. 


\section{Composition of the primaries}

Another signature to be sought is a statistical identification of the nature of the primaries as a function of their energies. Three methods have been identified to distinguish photon-initiated showers from hadron-initiated showers. One method relies on the longitudinal profile of the event, particularly the depth at shower maximum $x_{\max }$. The longitudinal profile of the Fly's Eye event at $3 \times 10^{20} \mathrm{eV}$ (well-measured by its nitrogen fluorescence trail) is ill-fit by a photon primary, well-fit by an iron primary, and somewhat fit by a proton primary [69]. The second method relies on measurement of muon number, with a high muon number purportedly favoring a hadron primary over a photon, and vice versa. A recent study of the muon content of showers above $10^{19} \mathrm{eV}$ seems to favor nucleons over photons [70]. However, caution is warranted with this method, in that some simulations show little difference in the muon-content of showers from nucleon primaries versus photon primaries [71]; and photons have themselves a significant partonic component at high energy. Nevertheless, it is safe to say that photon primaries appear disfavored at present, but more data is needed before conclusions are drawn.

The third method of gamma identification will will rely on a predicted characteristic N-S vs. E-W gamma asymmetry. This quadrupole asymmetry results from the polarization-dependent interaction of the gammas with the earth's magnetic field.

It is interesting to mention that the highest-energy Yakutsk event has an unusually high muon number; the only model so far which successfully explains this invokes a magnetic baryonic-monopole as the primary particle [31]. Basically, a relativistic baryonic-monopole of mass $M$ and incident energy $E$ showers like a giant nucleus of atomic number $A_{\text {eff }} \sim M / \Lambda_{\mathrm{QCD}} \sim 10^{6}(M / \mathrm{PeV})$ and the same $\gamma=E / M$. Intermediate mass monopoles therefore generate many, many charged pions which decay to muons.

All models wherein the primaries arise from QCD jets produce many more pions than baryons. The neutral pions in turn produce gammas. In these models, the ratio of gammas to baryons is typically of order ten at the source. Even allowing for the shorter attenuation length of photons relative to nucleons, the measurement of the primary compositon for super-GZK events becomes an excellent discriminator for models with jetproducing sources. These models include $Z$-bursts, and decaying SMPs and TDs.

\section{E. Possible $E_{\max }$ energy cutoff}

The predicted of a cutoff at $E_{\mathrm{GZK}}$ is wrong. Does Nature provide an alternative cutoff within our reach? Or do the data continue beyond our reach? The shock-jock experts claim that it is difficult for conventional shockacceleration mechanisms to produce $\mathrm{ZeV}$ proton energies; for this class of model, an $E_{\text {max }}$ below $10^{22} \mathrm{eV}$ is certainly expected [72]. Decaying SMP models also have a natural cutoff, at half of the SMP mass. This could be as high as $E_{\max } \sim 10^{24} \mathrm{eV}$ for a long-lived GUT-mass particle, but would be lower for other postulated SMPs. In the $Z$-burst model there is a natural cutoff related to the tiny mass of neutrinos: $E_{\max }=4\left(\mathrm{eV} / m_{\nu}\right) \mathrm{ZeV}$. Implications from the atmospheric SK data are that this cutoff is at most $7 \times 10^{22} \mathrm{eV}$.

\section{F. CR flux above vs. below $E_{\mathrm{GZK}}$}

A "smoothness" variable such as $R_{j} \equiv F_{j}(E>$ $\left.E_{\mathrm{GZK}}\right) / F_{j}\left(E<E_{\mathrm{GZK}}\right)$ for each primary species $j=$ nucleon, photon, iron nuclei, neutrino, etc. may be revealing [73]. For primaries with a GZK cutoff, $F(E<$ $\left.E_{\mathrm{GZK}}\right)$ samples sources from the whole volume of the Universe, and may even include cascade products from $F\left(E>E_{\mathrm{GZK}}\right)$, whereas $F\left(E>E_{\mathrm{GZK}}\right)$ samples just the GZK volume; for primaries without a GZK cutoff, $F\left(E<E_{\mathrm{GZK}}\right)$ and $F\left(E>E_{\mathrm{GZK}}\right)$ sample sources from the whole volume of the Universe. Lumps, bumps, and gaps in the spectrum near $E_{\mathrm{GZK}}$ are a consequence of some models. For example, hadron and neutrino pile-ups just below $E_{\mathrm{GZK}}$ are expected from the photo-pion production process which occurs above $E_{\mathrm{GZK}}[74]$. Other sources of neutrino pile-ups have also been suggested $[36,75]$. Present data show continuity. Smoothness studies of various models require simulation, and are just beginning.

\section{G. Spectral index above $E_{\mathrm{GZK}}$}

One means of achieving more events above $E_{\mathrm{GZK}}$ is to postulate a flattening of the primary proton spectrum at highest energies. With more data, the extreme-energy spectrum will be measured. A flattening of the $E^{-2.7}$ power law inferred from just below $E_{\mathrm{GZK}}$ would indicate and constrain new sources.

\section{H. Measureable neutrino flux above $E_{\mathrm{GZK}}$}

If there is a new source of primary neutrinos above $E_{\mathrm{GZK}}$, or if the neutrino cross-section becomes strong at super-GZK energies, then there is the possibility that the primary neutrino flux can be measured in an EUSO/OWL/AW-sized detector. This possiblity was discussed in section III D. 


\section{Diffuse $\sim \mathrm{GeV}$ gamma-ray flux}

An upper limit on the diffuse gamma-ray flux between $30 \mathrm{MeV}$ and $100 \mathrm{GeV}$ has been published by the EGRET experiment of the now defunct Gamma Ray Observatory [48]. This limit has serious discriminatory power for models where SMPs or TDs or extremely boosted massive particles decay to quark-antiquark jets which then hadronization to produce the EECRs [76]. This is because QCD jets via $\pi^{0}$ production and decay produce very high energy gammas which initiate an electromagnetic cascade on the cosmic radio, microwave, infrared, and magnetic field backgrounds. For models with jetproduction distributed over cosmic distances, such as some TD models and the $Z$-burst model with a homogeneous distribution of relic neutrino targets, the cascade has the distance to reach completion; the end result is gamma power (energy/time) in the EGRET range roughly an order of magnitude below the total power of the original sources, and comparable to the power in EECR neutrinos. Such models are disfavored. More local models, such as SMPs bound to our halo, and the $Z$-burst model with a local over-density of relic neutrinos, are not impacted at present, but may be tested with GLAST, the next generation gamma-ray observatory.

\section{CONCLUSIONS}

The ultimate explanation for the puzzles in EECRs will provide a surprise at a minimum, and possibly radically new physics at a maximum. The extreme energies of events already observed cannot be approached by terrestrial accelerators. Thus, there is ample motivation to build the next generation of CR detectors, Auger and Telescope Array, and to plan even farther beyond for a teraton detector like EUSO, OWL and AirWatch. At $10^{20} \mathrm{eV}$, AGASA provides about an event per year, and HiRes about an event per month. Auger and TA will see two such events per week, while EUSO/OWL/AW has the potential to collect such an event every two hours. As the data sample grows, statistical studies will reveal signatures that discriminate among the many galactic and extragalactic sources so far proposed to resolve the EECR puzzles.

It is quite possible that neutrino primaries are responsible for the EECRs. If so, it appears that the weaklyinteracting neutrino either grows a very strong crosssection at $10^{20} \mathrm{eV}$, or it annhilates on the relic neutrinos left-over from the hot phase of the Big Bang. Another possibility is that free magnetic charges exist and are the EECR primaries; for magnetic monopoles, the Universe is transparent and cosmic magnetic fields provide a natural acceleration mechanism.

Clearly, we live in exciting EECR times, and we possess the technology to prove this is so. The resolution of our puzzles is forthcoming, as on-going and future exper- iments will provide us with the statistics to discriminate among the many interesting models.

\section{ACKNOWLEDGEMENTS}

We acknowledge fruitful collaboration with P. Biermann, H. Goldberg, T. Kephart, H. Paes, and Dipthe Wick on some of the material presented here; and discussions with G. Farrar, G. Gelmini, A. Kusenko, D. McKay, A. Olinto, J. Ralston, G. Sigl, F. Stecker, and E. Zas. This work was supported in part by the U.S. Department of Energy grant no. DE-FG05-85ER40226.

[1] Recent reviews of EECR data, puzzles, and models include: P. Biermann, J. Phys. G23, 1 (1997); P. Bhattacharjee and G. Sigl, Phys. Rept. 327, 109 (2000) [astroph/9811011]; A.V. Olinto, "David Schramm Memorial Volume" of Phys. Rept. 333, 329 (2000); X. Bertou, M. Boratov, and A. Letessier-Selvon, Int. J. Mod. Phys. A15, 2182 (2000); A. Letessier-Selvon, Lectures at "XXVIII International Meeting on Fundamental Physics", Cadiz, Spain (2000) [astro-ph/0006111]; M. Nagano and A.A. Watson, Rev. Mod. Phys. 72, 689 (2000); A. Olinto, astro-ph/0011106; M. Kachelriess, astro-ph/0011231; G. Sigl, Science 291, 73 (2001); F.W. Stecker, astro$\mathrm{ph} / 0101072$.

[2] M. Takeda et al. (AGASA Collaboration), Phys. Rev. Lett. 81, 1163 (1998) [astro-ph/9807193]; D.J.Bird et al. (Fly's Eye Collaboration), Astrophys. J. 424, 491 (1994), and ibid 441, 144 (1995); M.A. Lawrence, R.J.O.Reid and A.A. Watson (Haverah Park Collaboration), J. Phys. G 17,773 (1991), and M. Ave et al., Phys. Rev. Lett. 85, 2244 (2000); N.N. Efimov et al. (Yakutsk Collaboration), Proc. "Astrophysical Aspect of the Most Energetic Cosmic Rays," p. 20, eds. M. Nagano and F. Takahara, World Sci., Singapore, 1991; D. Kieda et al. (HiRes Collaboration), Proc. of the 26th ICRC, Salt Lake City, Utah, 1999.

[3] J. Elbert and P. Sommers, Astrophys. J. 441, 151 (1995).

[4] Named after the pioneering work of Greisen, Kuzmin, and Zatsepin in the 1960's; recent detailed explorations of the GZK cutoff include S. Lee, Phys. Rev. D58, 043004 (1998); A. Achterberg et al., astro-ph/9907060; T. Stanev et al., astro-ph/0003484.

[5] A.M. Hillas, Ann. Rev. Astron. Astrophys. 22, 425 (1984); C.A. Norman, D.B. Melrose, and A. Achterberg, AStrophys. J. 454, 60 (1995); R.D. Blandford, Physica Scripta T85, 191 (2000).

[6] N. Hayashida et al. (AGASA Collaboration), Phys. Rev. Lett. 77, 1000 (1996); M. Takeda et al., Astrophys. J. 522, 225 (1999) [astro-ph/9902239 and astro-ph/0008102].

[7] Teshima, reported at "Radio Detection of High Energy 
Neutrinos 2000", UCLA, Nov. 2000, ed. P. Gorham and D. Saltzberg.

[8] Uchihori et al., Astropart. Phys. 13, 151 (2000) [astro$\mathrm{ph} / 9908193]$.

[9] P.L. Biermann and G.R.Farrar, Phys. Rev. Lett. 81, 3579 (1998); ibid 83, 2478(E) (1999).

[10] G.Sigl, D.F.Torres, L.A.Anchordoqui and G.E.Romero, astro-ph/0008363; A. Virmani et al., atro-ph/0010235.

[11] H. Goldberg and T.J. Weiler, astro-ph/0009378.

[12] Websites for existing and proposed large-area CR experiments are:

http://ast.leeds.ac.uk/haverah/hav-home.shtml for Haverah Park;

http://www-akeno.icrr.u-tokyo.ac.jp/AGASA/ for

AKENO;

http://hires.physics.utah.edu for HiRes;

http://www.auger.org

http://www-lpnhep.in2p3.fr/auger/welcome.html

for Auger;

http://www-ta.icrr.u-tokyo.ac.jp/

for Telescope Array;

http://ifcai.pa.cnr.it/ifcai/euso.html

for EUSO;

http://owl.gsfc.nasa.gov for OWL;

http://ifcai.pa.cnr.it/ AirWatch/

for AirWatch.

[13] W. Springer and J. Belz, private communication.

[14] E. Waxman, K.B. Fisher and T. Piran, Astrophys. J. 483,1 (1997); M. Lemoine, G. Sigl, A. Olinto and D.N. Schramm, Astrophys. J. 486, L115 (1997); G. Sigl, M. Lemoine and A. Olinto, Phys. Rev. D56, 4470 (1997); G. Sigl and M. Lemoine, Astropart. Phys. 9, 65 (1998); G.A.Medina-Tanco, Astrophys. J. L71, 495 (1998), and astro-ph/9707054; V. Berezinsky, hep-ph/0001163; S.L.Dubovsky, P.G.Tinyakov and I.I.Tkachev, Phys. Rev. Lett. 85, 1154 (2000); Z.Fodor and S.D.Katz, hep$\mathrm{ph} / 0007158$.

[15] Topological Defect physics is reviewed in A. Vilenkin and E.P.S. Shellard, Cosmic Strings and Other Topological Defects, Cambridge Pr., 1994; M.B. Hindmarsh and T.W.B. Kibble, Rep. Prog. Phys. 58, 477 (1995).

[16] C.T. Hill, Nucl. Phys. B224, 469 (1983); J.J. BlancoPillado and K.D. Olum, Phys. Rev. D60, 083001 (1999).

[17] A review is given in V. Kuzmin and I. Tkachev, Phys. Rept. 320, 199 (1999).

[18] K. Hamaguchi, Y. Nomura, and T. Yanagida, Phys. Rev. D58, 103503 (1998); K. Hamaguchi, K.I. Izawa, Y. Nomura, and T. Yanagida, Phys. Rev. D60, 125009 (1999).

[19] J. Ellis, J.L. Lopez, and D.V. Nanopoulos, Phys. Lett. B247, 257 (1990); K. Benakli, J. Ellis, and D.V. Nanopoulos, Phys. Rev. D59, 047301 (1999).

[20] V. Berezinsky, M. Kachelriess, and A. Vilenkin, Phys. Rev. Lett. 79, 4302 (1997).

[21] V.A. Kuzmin and V.A. Rubakov, Phys. Atom. Nucl. 61, 1028 (1998).

[22] S.L. Dubovsky and P.G. Tinyakov, JETP Lett. 68, 107 (1998); V. Berezinsky, P. Blasi, and A. Vilenkin, Phys. Rev. D 58, 103515 (1998).

[23] E-J Ahn, G. Medina-Tanco, P.L. Biermann, T. Stanev, astro-ph/9911123.
[24] M. Hillas, Nature 395, 15 (1998); G. Farrar and T. Piran, astro-ph/0010370; L.A. Anchordoqui, H. Goldberg, and T.J. Weiler, in progress.

[25] F.W. Stecker, Astropart. Phys. 14, 207 (2000).

[26] G.R.Farrar, Phys. Rev. Lett. 76, 4111 (1996); D.J.H.Chung, G.R.Farrar and E.W.Kolb, Phys. Rev. D57, 4606 (1998); I.F.M. Albuquerque, G.R. Farrar and E.W. Kolb, Phys. Rev. D59, 015021 (1999).

[27] N.A. Porter, Nuovo Cim. 16, 958 (1960); E. Goto, Prog. Theo. Phys. 30, 700 (1963).

[28] T.W. Kephart and T.J. Weiler, Astropart. Phys. 4, 271 (1996).

[29] S.D. Wick, T.W. Kephart, T.J. Weiler and P.L. Biermann, Astropart. Phys. (to appear) [astro-ph/0001233].

[30] A. S. Goldhaber, in Phys. Rept. 315, 83 (1999) and [hepth/9905208]; an unrelated idea for a strong monopole cross-section is given in E. Huguet and P. Peter, Astropart. Phys. 12, 277 (2000).

[31] L. Anchordoqui et al., hep-ph/0009319.

[32] C.O. Escobar and R.A. Vazquez, Astropart. Phys. 10, 197 (1999).

[33] Mestres-Gonzales, Proc. 25th ICRC, 1997, Durban, So. Africa, (World Sci., Singapore), and Workshop on Observing Giant CR Air-Showers from $>10^{20} \mathrm{eV}$ Particles from Space, ed. J.F. Krizmanic et al., 1998 (AIP Conf. Proc. No. 433, Woodbury, NY); S. Coleman and S.L. Glashow, hep-ph/9808446, and Phys. Rev. D59, 116008.

[34] Y.J. Ng, D.S. lee, M.C. Oh, and H. van Dam, hep$\mathrm{ph} / 0010152$.

[35] T.J. Weiler, Phys. Rev. Lett. 49, 234 (1982); Astrophys. J. 285, 495 (1984); E. Roulet, Phys. Rev. D47, 5247 (1993);

[36] T.J. Weiler, Astropart. Phys. 11, 303 (1999), and ibid. $12,379 \mathrm{E}$ (2000) [for corrected receipt date].

[37] D. Fargion, B. Mele and A. Salis, Astrophys. J. 517, 725 (1999).

[38] G. Gelmini and A. Kusenko, Phys. Rev. Lett. 82, 5202 (1999).

[39] Review of Particle Physics, Euro. Phys. J. C15, 1 (2000).

[40] H. Paes and T.J. Weiler, Phys. Rev. D, to appear [hep$\mathrm{ph} / 0101091]$.

[41] S. Yoshida, G. Sigl, and S. Lee, Phys. Rev. Lett. 81, 5505 (1998); G. Gelmini, hep-ph/0005263.

[42] V. Barger, T.J. Weiler, and K. Whisnant, Phys. Lett. B442 255 (1998).

[43] Reviewed by H. Sobel (SuperK Collaboration), at Neutrino 2000, Sudbury, Canada, June 2000.

[44] Reviewed by G. Mills (LSND Collaboration), at Neutrino 2000, Sudbury, Canada, June 2000.

[45] P. Gorham, K. Liewer, and C. Naudet, [astroph/9906504] and Proc. 26th Int. CR Conf., Salt Lake City, Utah, Aug. 1999.

[46] J.J. Blanco-Pillado, R.A. Vazquez, and E.Zas, Phys. Rev. D61, 123003 (2000).

[47] S. Yoshida, G. Sigl, and S. Lee, in [41].

[48] P. Sreekumar et al., Astrophys. J. 494, 523 (1998).

[49] G. Gelmini and A. Kusenko, Phys. Rev. Lett. 84, 1378 (2000); J.L. Crooks, J.O. Dunn, and P.H. Frampton, astro-ph/0002089.

[50] C. Tyler, A.V. Olinto, and G. Sigl, hep-ph/0002257. 
[51] V.S. Berezinsky and G.T. Zatsepin, Phys. Lett. 28B, 423 (1969); G. Domokos and S. Nussinov, Phys. Lett. B 187, 372 (1987);

[52] J. Bordes et al., hep-ph/9705463; Astropart. Phys. 8, 135 (1998).

[53] G. Domokos, S. Kovesi-Domokos, and P.T. Mikulski, hep-ph/0006328.

[54] S. Nussinov and R. Shrock, Phys. Rev. D59, 105002 (1999); G. Domokos and S. Kovesi-Domokos, Phys. Rev. Lett. 82, 1366 (1999); P. Jain, D.W. McKay, S. Panda and J.P. Ralston, Phys. Lett. B484, 267 (2000).

[55] H. Goldberg and T. J. Weiler, Phys. Rev. 59, 113005 (1999).

[56] M. Kachelriess and M. Plumacher, Phys. Rev. D62, 103006 (2000); L. Anchordoqui et al., hep-ph/0011097.

[57] A. Jain, P. Jain, D.W. McKay, and J.P. Ralston, hepph/0011310.

[58] F. Cornet, J.I. Illana, and M. Masip, hep-ph/0102065.

[59] P.P. Kronberg, Rep. Prog. Phys. 57, 325 (1994); J.P. Vallee, Fund. Cosmic Phys. 19, 1 (1997); P. Blasi, S. Burles, and A.V. Olinto, Astrophys. J. 514, L79 (1999).

[60] There is new evidence for large $\mu \mathrm{G}$ magnetic fields filling the space within galaxy clusters: T.E. Clarke, P.P Kronberg, and H. Boehringer, astro-ph/0011281.

[61] V. Berezinsky, M. Kachelriess and A. Vilenkin, Phys. Rev. Lett 79, 4302 (1997); V.A.Kuzmin and V.A.Rubakov, Phys. Atom. Nucl. 61, 1028 (1998); M. Birkel and S. Sarkar, Astropart. Phys. 9, 297 (1998); P.Blasi, Phys. Rev. D60, 023514 (1999); S. Sarkar, hepph/0005256.

[62] P. Bhattacharjee, C. Hill, and D. Schramm, Phys. Rev. Lett. 69, 567 (1992); G. Sigl, S. Lee, P. Bhattacharjee, and S. Yoshida, Phys. Rev. D59, 043504 (1999).

[63] P. Blasi and R. Sheth, Phys. Lett. B486, 233 (2000).

[64] G. Sigl, M. Lemoine, and P. Biermann, Astropart. Phys. 10, 141 (1999), and astro-ph/9903124; D.Harari, S. Mollerach and E. Roulet, J. High Energy Phys. 08, 022 (1999); S. Mollerach and E. Roulet, astro-ph/9910205.

[65] T. Stanev et al., Phys. Rev. Lett. 75, 3056 (1995); G. Medina-Tanco, Astrophys. J. 510, 91 (1999); J. Bahcall and E. Waxman, hep-ph/9912326; O.E. Kalashev, V.A. Kuzmin, and D.V. Semikoz, astro-ph/0006349; M. Blanton, P. Blasi, and A. Olinto, astro-ph/0009466.

[66] P. Blasi and R.K.Sheth, astro-ph/0006316.

[67] A. Kusenko and V.A. Kuzmin, astro-ph/0012040.

[68] D.Harari, S. Mollerach and E. Roulet, J. High Energy Phys. 02, 035 (2000).

[69] F. Halzen, R.A. Vazquez, T. Stanev, and H.P. Vankov, Astropart. Phys. 3, 151 (1995); M. Nagano and A.A. Watson, fig. 16 by Heck, in [1]; Ave et al., in [2]; T.K.Gaisser (figure courtesy of T. Stanev), Proc. International Workshop on Observing Ultra-High Energy Cosmic-Rays from Space and Earth, Metepec, Puebla, Mexico, Aug. 9-12, 2000, ed. A. Zepeda et al.

[70] M. Ave, J.A. Hinton, R.A. Vazquez, A.A. Watson, and E. Zas, Phys. Rev. Lett. 85, 2244 (2000).

[71] F.A. Aharonian, B.L. Kanevsky, and V.A. Sahakian, J. Phys. G17, 1909 (1991).

[72] See, e.g., the review of P. Biermann in ref. [1], and of R. Blandford in ref. [5].
[73] Discussed in Farrar and Piran [24].

[74] J.L. Puget, F.W. Stecker, and J. Bredkamp, Astrophys. J. 205, 638 (1976); C.T. Hill and D.N. Schramm, Phys. Rev. D31, 564 (1985); V.S. Berezinsky and S.I. Grigoreva, Astron. Astrophys. 199, 1 (1988); also, T. Stanev et al. in [4].

[75] A. Kusenko and M. Postma, hep-ph/0007246; M. Postma, hep-ph/0102106.

[76] S. Lee; R.J Protheroe and P.A. Johnson, Nucl. Phys. B Proc. Suppl. 48, 485 (1996); R.J Protheroe and T. Stanev, Phys. Rev. Lett. 77, 3708 (1996); Sigl, Lee, Yoshida, in [?]. 